\title{
Genomics, epigenomics and chromatin
}

\author{
A. K. Sharma
}

Published online: 15 July 2011

(C) Archana Sharma Foundation of Calcutta 2011

In recent years, epigenetic expression and its control leading to complex manifestation of differentiation have been widely discussed. As is well known, genetics covers analysis of structure and behaviour of the entire genome, whereas epigenetics involves the study of inherited changes or gene expression in the lifetime of an individual without involving change of nucleic acid bases. Epigenetics in its totality and not in relation to mere individual trait, is termed epigenomics. According to certain authors [9] it may be denoted as extra-layer of gene control or genomic influence for maintenance of specific cell types and expression. The role of Epigenetics in evolution, though heritable in the somatic cells, is not as clear as Genetics, where the proved mechanism is variation and selection.

The epigenetic changes during gene expression cover different components of the nucleus, the DNA, the protein and the nucleosome at the chromosome level. The modification may involve methylation of DNA, modeling and positioning of nucleosome, change in histone pattern, small RNAs in general, non coding, mobile and micro RNAs, alteration of protein expression in a variety of ways but with specific targets at the transcriptional to post translational level. All these changes act in conjunction with transcription factors and regulators to effect gene expression in its totality. The role of repeated gene on ageing and cell proliferation is also exhibited.

The genomic DNA determines intrinsic stability of epigenetic event as it encodes nucleosome structure and positioning as well. The specificity and non random nature

A. K. Sharma $(\bowtie)$

Centre of Advanced Study in Cell and Chromosome Research,

Department of Botany, University of Calcutta,

Kolkata 700 019, India

e-mail: nuclaks@cal2.vsnl.net.in at the base level is often reflected in the results of their interaction, at specific sites which lead to silencing, activation, and acceleration. For example, acetylation of histone at the promoter site often leads to activation but at the coding site it results in repression [9]. Post transcriptional modification of histones usually involve terminal ends of core histone. Similarly DNA methylation is very conserved at the cytosine level, but at the promoter site it leads to repression [5]. In fact it is well known that tumor suppressor genes are suppressed by methylation. Highly dense GC regions show less methylation as compared to that of intermediate GC content $[1,2]$

The importance of DNA sequence in nucleosome positioning and stability is also indicated as the $5^{\prime}$ end is often preferred [7]. These are some of the examples where epigenetic tools like DNA methylation, histone positioning, protein modifications and non coding RNAs act in a predetermined fashion.

The epigenetic changes however, though lead to chromatin modification, it is often difficult to demonstrate, excepting in the behaviour of specific heterochromatic ones through molecular hybridization with specific probes at the microscopic level, as well as in dosage compensation of $\mathrm{X}$ chromosome in Drosophila. In the manifestation of chromatin modification, a very pertinent example is provided by CENPA - Kinetochore complex which is aided by RNAi induced heterochromatin but later perpetuates through epigenetic method without heterochromatin [4].

Several positive cases have been recorded in recent years on genetic control of the epigenetic phenomenon as reflected at the chromosome level. The position and organization of the eukaryotic nucleus and the dynamics of chromosomes in facilitating replication, transcription repair and recombination have all been clearly demonstrated through in situ labeling in molecular hybridization. The stability of chromosome territo- 
Fig. 1 Somatic chromosomes $(2 \mathrm{n}=20)$ of Cymbopogon winterianus showing in situ immuno-localization of $5 \mathrm{mC}$ methylation sites. a DAPI stained chromosome plate, $\mathbf{b}$ showing $5 \mathrm{mC}$ localization-red signals (picture by courtesy UC Lavania and Y Mukai)
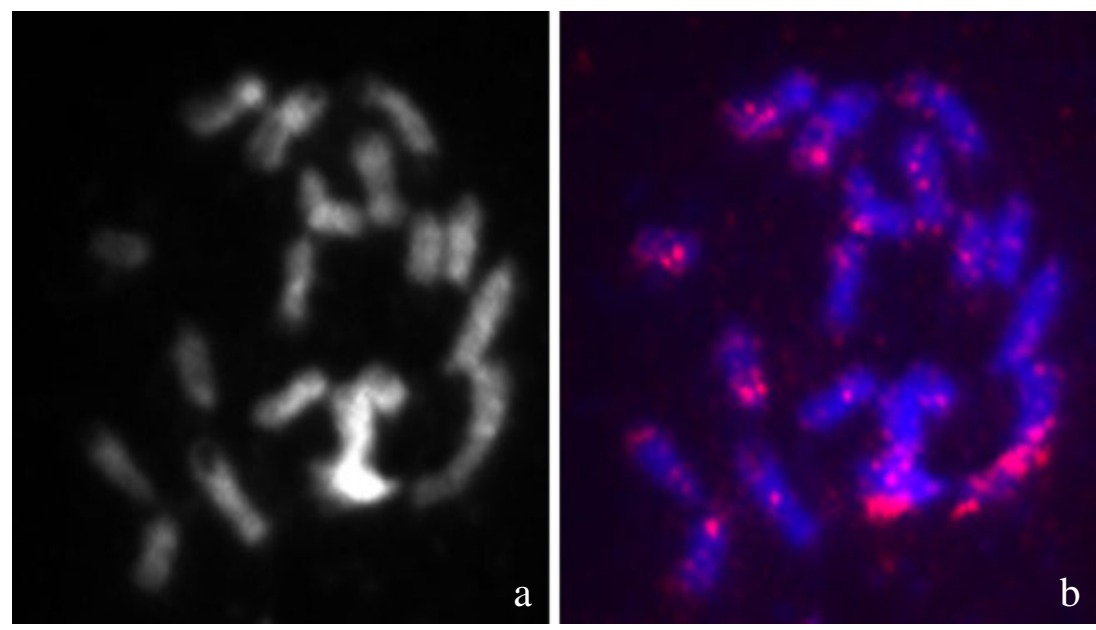

rial distribution in nucleus and shift during transcription is reiterated [6]. The changed cytosine methylation and consequent gene expression in induced Brassica allopolyploids is a case in point [8]. Similarly increased DNA methylation in autotetraploid as compared to diploid in the aromatic grass, Cymbopogon has been observed (Lavania, UC and Mukai Y, personal communication). More methylation of transgenes in autotetraploid Arabidopsis is also on record [3].

In addition to epigenetic events and genetic control, the behaviour of transposons as a part of programmed gene expression is often cited. But its sensitivity to stress and capacity to induce mutation make the issue highly debated.

The unquestionable link of epigenetic event with genetic control and its manifestation at the chromosomal level involves a complex relationship which is yet to be fully deciphered and the decoding of entire epigenome is being planned. In fact, this programme demands high priority in view of the stress prone epigenetic process, especially in the present decade and the decades to come when the climate change is on the anvil. A critical analysis of all the epigenetic events, their genetic control and their manifestation at the fine structural level of chromosome both condensed and decondensed wherever possible concomitant with gene expression is indeed called for (Fig. 1).

\section{References}

1. Das R, Dimitrova N, Xuan Z, Rollins RA, Haghighi F, Edwards JR, et al. Computational prediction of methylation status in human genomic sequences. Proc Natl Acad Sci USA. 2006;103:10713-6.

2. Feinberg AP, Irizarry RA. Stochastic epigenetic variation as a driving force of development, evolutionary adaptation and disease. Proc Natl Acad Sci USA. 2010;107 Suppl 1:1757-64.

3. Finn TE, Wang L, Smolilo D, Smith NA, White R, Chaudhury A, et al. Transgene expression and transgene-induced silencing in diploid and autotetraploid Arabidopsis. Genetics. 2011;187:409-23.

4. Folco HD, Pidoux AL, Urano T, Allshire RC. Heterochromatin and RNAi are required to establish CENP-A chromatin at centromeres. Science. 2008;319:94-7.

5. Lister R, Pelizzola M, Dowen RH, Hawkins RD, Hon G, TontiFilippini J. Human DNA methylomes at base resolution show widespread epigenomic differences. Nature. 2009;462:315-22.

6. Schubert I, Shaw P. Organization and dynamics of plant interphase chromosomes. Trends Plant Sci. 2011;16:273-81.

7. Segal E, Fondufe-Mittendorf Y, Chen L, Thastrom A, Field Y, Moore IK. A genomic code for nucleosome positioning. Nature. 2006;442:772-8

8. Xu Y, Zhong L, Wu X, Fang X, Wang J. Rapid alterations of gene expression and cytosine methylation in newly synthesized Brassica napus allopolyploids. Planta. 2009;229:471-83.

9. Yuan GC. Prediction of epigenetic target sites by using genomic DNA sequence. In: Liu LA, Wei D, Li Y, Lei H, editors. Handbook of research on computational and systems biology: interdisciplinary applications. 2011;1:187-201. IGI Global. doi:10.4018/978-1-60960491-2.ch008. 